\title{
Reactive changes of disc space and foreign body granuloma due to bone wax in lumbar spine
}

\author{
Nail Ozdemir, Mustafa Fazıl Gelal ${ }^{1}$, Mustafa Minoglu, Levent Celik \\ Departments of Neurosurgery and ${ }^{1}$ Radiology, Izmir Atatürk Training and Research Hospital, Izmir, Turkey
}

\begin{abstract}
Address for correspondence:
Dr. Nail Ozdemir,

Catalkaya M, Pir Sultan Abdal S,

No:42 D:7 35320, Narlıdere-Izmir,

Turkey.

E-mail: dr.nailozdemir@gmail.com
\end{abstract}

DOI: $10.4103 / 0028-3886.55606$

\begin{abstract}
Bone wax is a well-known agent used to prevent bleeding from the bone that can be used in different surgical procedures. Bone wax is a safe agent, but it may rarely lead to significant foreign body reactions. In this report, we present a patient who developed bone wax-related disc space foreign body granuloma following L4 total laminectomy, extremely rare complication.
\end{abstract}

Key words: Bone wax, disc space changes, foreign body granuloma, lumbar disc surgery, spinal surgery

\section{Introduction}

Bone wax is extensively used to control bleeding from bony structures in various surgical procedures. Although bone wax is a safe agent, it may rarely lead to significant complications, such as compression of dural sac and root with foreign body granuloma after lumbar disc surgery ${ }^{[1]}$ cervical epidural compression, ${ }^{[2]}$ intracranial foreign body granuloma, ${ }^{[3,4]}$ recurrent epistaxis, ${ }^{[5]}$ sigmoid sinus trombosis, ${ }^{[6]}$ calvarial granulomatous infection and fistula, ${ }^{[7]}$ orbital granulomatous infection, ${ }^{[8]}$ retroperitoneal tumor ${ }^{[9]}$ and saphenous vein graft thrombosis. ${ }^{[10]}$ In this report, we present a case with reactive disc space changes and foreign body granuloma in L4 total laminectomy area due to remnant bone wax after lumbar spinal surgery.

\section{Case Report}

A 39-year-old man was admitted to our institution for persistent severe pain in his lower back. He had had a history of lumbar surgery for spinal stenosis at L4-L5 level ten months earlier, including L4 total laminectomy, L4-5 discectomy, and bilateral L5 foraminotomy. The patient had an L4-5 discectomy, and did not have the endplates curetted. Postoperatively, the patient noticed that his leg pain and numbness had improved, but his lower back pain had gradually increased. The patient had been initially treated with nonsteroidal antiinflammatory tablets and continuing physiotherapy, but his discomfort became worse and his incapacity more severe. On examination, his spine was straight, and there was tenderness to palpation over the surgical area, with limitation of lumbar movements. Straight leg raising test was negative. There were no signs of neurological deficit.

Lumbar magnetic resonance imaging (MRI) scans showed contrast enhancement at L4-5 disc space representing discitis; and a 2 -cm cystic lesion at the site of the resected spinous process with contrast enhancing margins representing probably an abscess [Figure 1a-d]. Laboratory investigation was as follows:Hemoglobin $15.0 \mathrm{~g} / \mathrm{dl}$, WBC $14.800 / \mathrm{mm}^{3}$ with differential count. The erythrocyte sedimentation rate (ESR) was $7 \mathrm{~mm} / \mathrm{h}$ and $C$ reactive protein $(\mathrm{CRP}) 0.5 \mathrm{mg} / \mathrm{L}$. In the operation; a 20-millimeter lesion with yellow-beige color and a smooth surface that could be separated easily from the surrounding tissues at the site of the resected spinous process was encountered [Figure 2]. When the lesion was taken out, we noticed that bone wax was excessively smeared to the edge of the right facet and that there was a residual bone wax fragment separate from the bone, several millimeters in length. This residual bone wax fragment, located next to the right facet joint, away from 

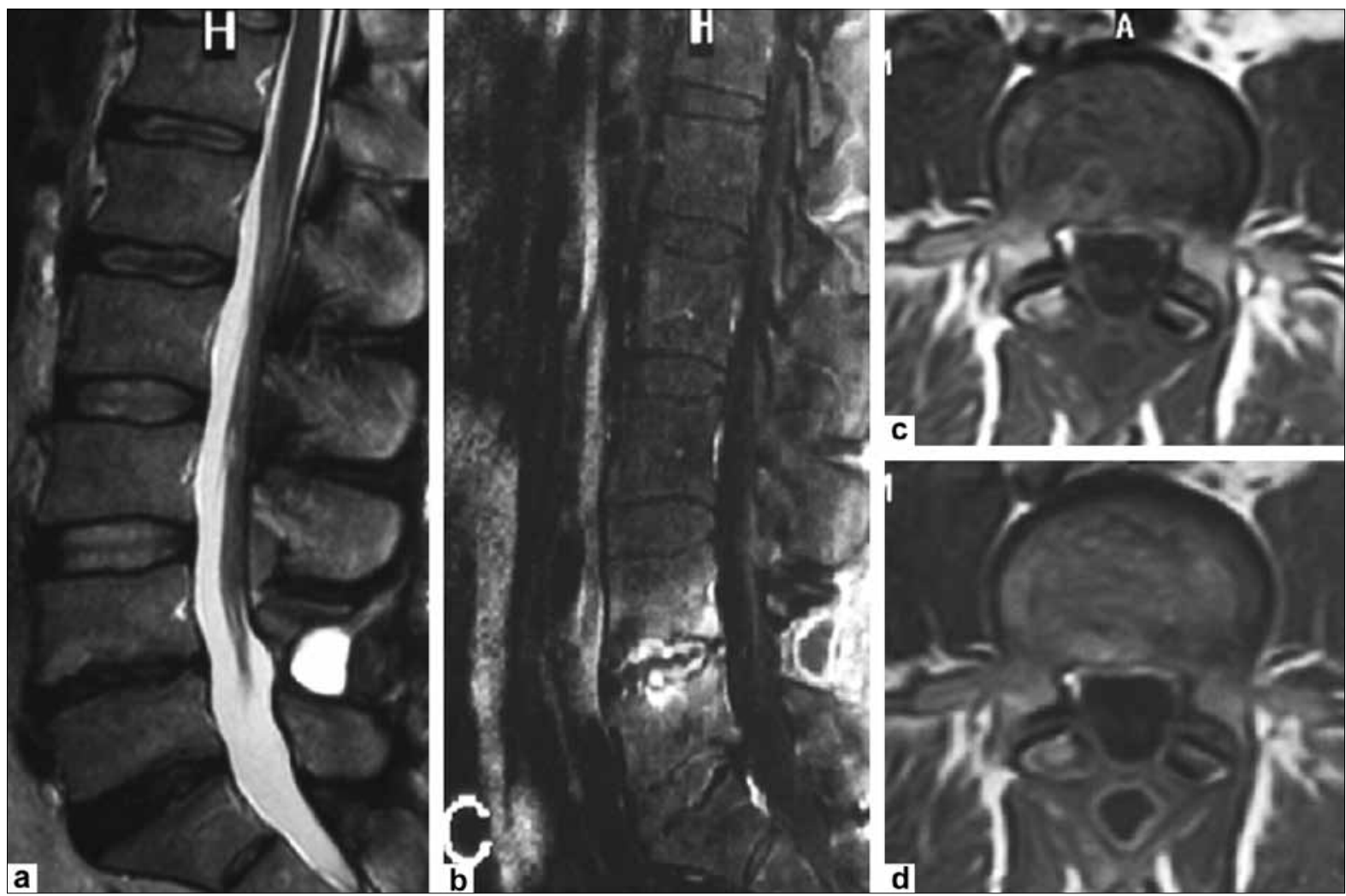

Figure 1: Reactive changes of disc space and foreign body granuloma after spinal surgery. (a) T2 weighted sagittal MRI scan shows a cystic lesion at the site of resected spinous process and loss of height and signal in L4-5 disc; (b) Fat saturated post-contrast T1-weighted sagittal scan shows

that the periphery of the cystic lesion and the surrounding tissue is enhancing. Also note the contrast enhancement of L4-5 disc, representing probably triggered by discitis or unknown inflammatory agent. Precontrast; (c) postcontrast (d); T1-weighted axial scans demonstrate the peripherally enhancing cystic lesion at the site of operation

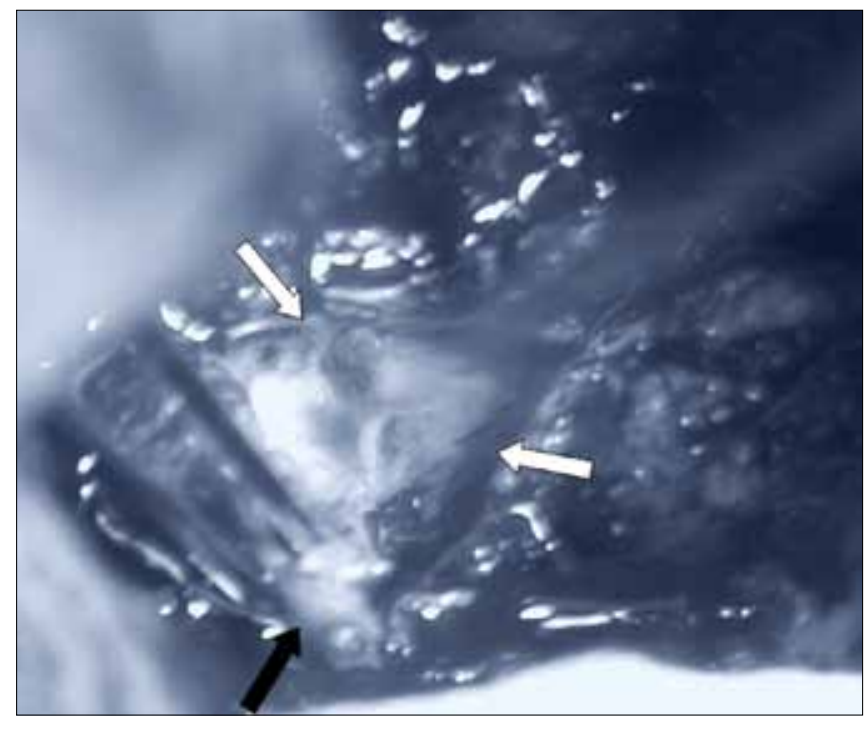

Figure 2: Intraoperative photograph. A 20-millimeter lesion and a smooth surface was noted at the laminectomy area (white arrows). The lesion is held up with tissue forceps (black arrow). When the lesion was taken out, we noticed that residual bone wax fragment was separate from the edge of the right facet

the disc space was also removed. The lesion was sent to microbiology for culture. Culture of the lesion yielded no pathogens. The histopathological examination revealed foreign body granuloma [Figure $3 a$ and $b$ ].
The postoperative course was uneventful. He had complete relief form his persistent lower back pain. The patient was discharged on the fifth postoperative day. A postoperative MRI was performed ten months later which revealed no lesions in both L4-L5 disc space and laminectomy area [Figure $4 \mathrm{a}$ and $\mathrm{b}$ ].

\section{Discussion}

Bone wax is an unresorbable mixture of beeswax $(70 \%)$ and vaseline (\%30). Covering the bone borders with bone wax provides a physical barrier to the opened intra-osseous vessel canals. ${ }^{[11]}$ It is a well-known agent used to prevent bleeding from the bone that can be used both in elective and traumatic spinal surgery practice.

There are only a limited number of studies which have investigated the foreign body reactions of bone wax. Eser et al., ${ }^{[12]}$ reported that the excessive use of bone wax in lumbar spine surgery caused a foreign body reaction, as well as direct compression of the dural sac and spinal root on MRI. Cirak and Unal ${ }^{[2]}$ reported a case of iatrogenic quadriplegia, which was related to either direct compression from bone wax or epidural bleeding caused by detachment of epidural veins due to 


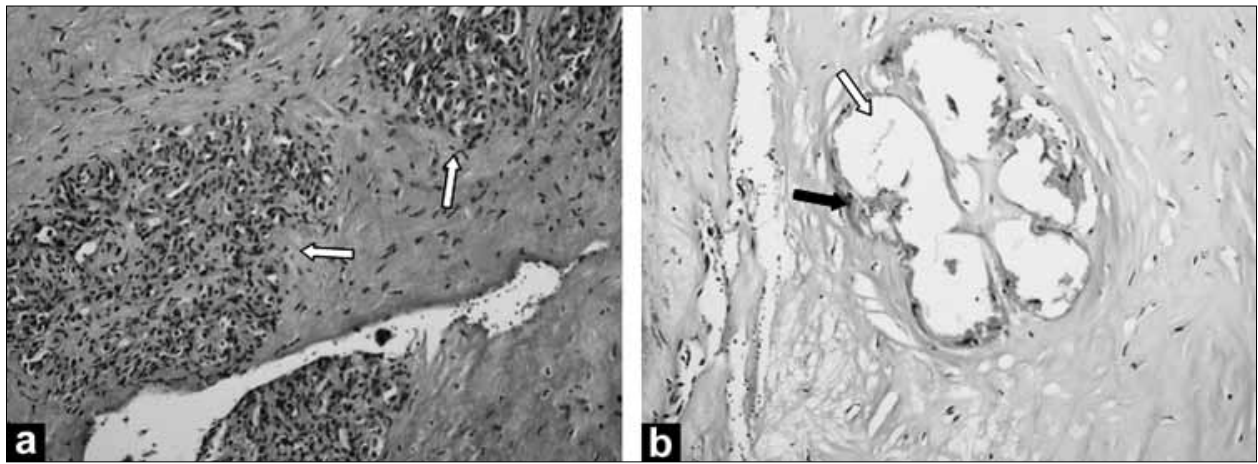

Figure 3: Photographs of histological examination in our case. (a) $\mathrm{H}$ and $\mathrm{E}, \times 100$, capillary vascular proliferation, minimally mononuclear cell infiltration and fibroblasts in fibrous tissue (white arrows); (b) $\mathrm{H}$ and $\mathrm{E}, \times 400$, the foreign body (white arrow) and multinuclear benign giant cell (black arrow)
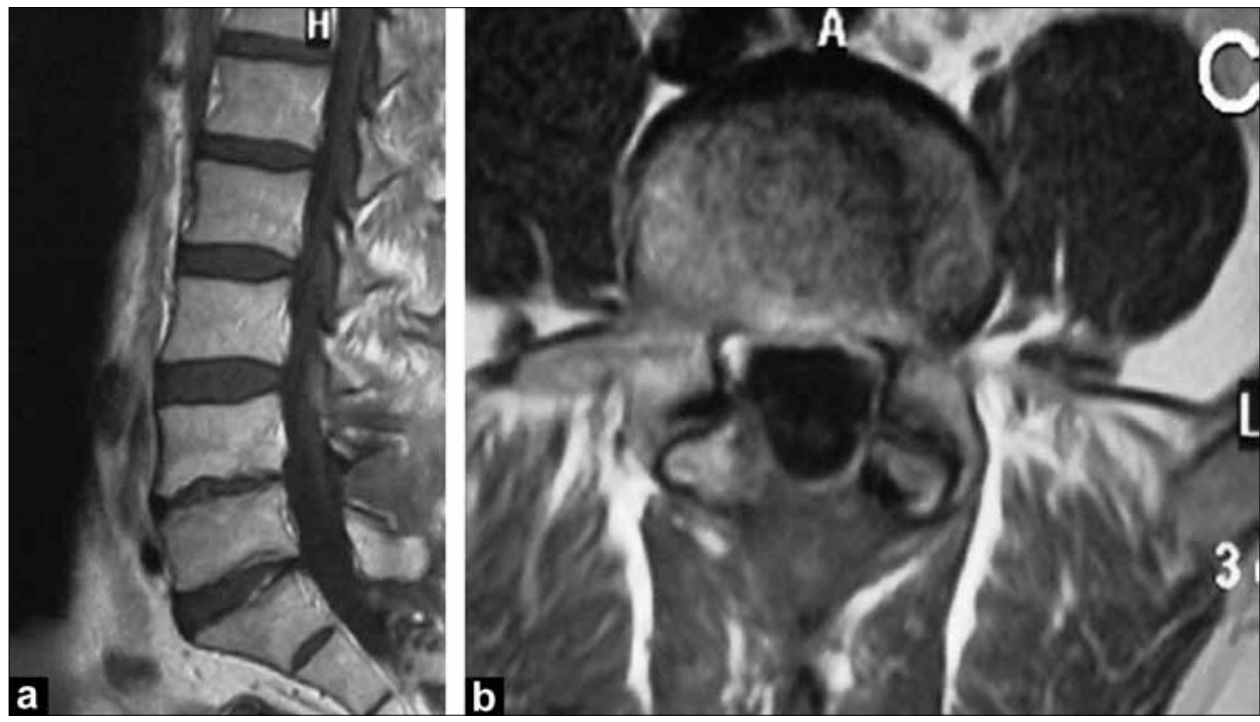

Figure 4: The follow-up MRI at 10 months after second operation. Post-contrast T1-weighted sagittal; (a) axial; (b) scans show that both the granuloma and changes of disc space at L4-L5 level disappeared

irritating effect of bone wax. Ates et al., ${ }^{[3]}$ reported a case presenting with foreign body granuloma infiltration of the medulla oblongata caused by remnant bone wax in the subarachnoid space after posterior fossa decompressive surgery. Patel et al., ${ }^{[4]}$ reported that the enhancement inside the cerebellar parenchyma near the remnant of bone wax might be due to reactive inflammatory response of neural tissue to the foreign body. Wolvius and van der $\mathrm{Wal}^{[7]}$ reported a case of foreign body granuloma caused by bone wax in a cranial defect in the parietal region. Sudmann et al., ${ }^{[13]}$ demonstrated with anatomicopathological study of 18 cadavers that bone wax is an unresorbable hemostatic bone sealant which induces chronic inflammation in the sternum postoperatively and computed tomography failed to detect bone wax-related granuloma in cadaver sterna. Seven cases of bone wax granuloma have been reported by Anfinsen et al., ${ }^{[14]}$ in women who underwent foot surgery. At re-operation, soft granulation tissue was resected. Microscopically, in all cases, it can induce chronic inflammation and marked foreign body reaction after application in the calcaneus. Sorrenti et al. ${ }^{[15]}$ investigated the responses of the human tibias to bone wax. They found a definite foreign body giant cell reaction to bone wax in 12 patients treated by elevation of the tibial tubercules. Giant cells eliminated bone wax particules from the site, culminating in a fibrous reaction; inflammatory changes were seen after six to nine months. The samples that were obtained at 13 months clearly demonstrated a fibrous reaction. Verborgt et al., ${ }^{[10]}$ reported a case of a retroperitoneal tumor as a late complication of the use of bone wax in harvesting iliac crest. The tumor needed surgical removal 19 years later. Microscopically, a bone wax granuloma was diagnosed. Effects of bone wax on bacterial clearance have been studied by Johnson and Fromm ${ }^{[16]}$ in iliac crest of rabbits. They showed that bone wax significantly reduced the ability of cancellous bone of rabbits to clear bacteria. Microfibrillar collagen, bone wax, gelatin foam and oxidized regenerated cellulose from topical hemostatic agents were compared by Finn et al., ${ }^{[17]}$ concerning their effects on osseous regeneration of the iliac crest of four dogs. Bone wax showed a marked foreign body reaction and lack of bone reformation. They concluded that microfibrillar collagen, oxidized regenerated cellulose, and gelatin foam might be 
adequately used in iliac bone procurement, whereas bone wax seems to be contraindicated.

Silber et al., ${ }^{[18]}$ had reported a review of the literature on the subject of postprocedural discitis in 2002. As a result of searching the literature about this entity, some authors have described two different types of postprocedural discitis, as following (a) septic discitis (b) aseptic discitis. A septic form was caused by an infectious agent and an aseptic form resulted from an inflammatory reaction. Contrast enhancement is highly suggestive of hypervascularity caused by the inflammatory process triggered by the infection. The abscess formation (purulent material) is usually depicted by a high-intensity signal on T2 MRI and low-intensity signal on T1. The peripheral ring represents granulation tissue and the central zone fluid that may suggest abscess formation.

Reactive changes of disc space and foreign body granuloma due to remnant bone wax after lumbar spinal surgery has never been presented before in the literature. The preoperative MRI diagnosis for the cystic lesion located at the site of resected spinous process was abscess. However, in the operation we found a granuloma and bone wax remnants, rather than an abscess. The granuloma was probably induced by the remnants of bone wax and both were removed. We noticed that bone wax was excessively smeared to the edge of the right facet and was not related to the L4-5 disc space. We deliberated that changes of L4-5 disc space might be reactive inflammatory response due to remnant bone wax in the operation. Therefore, he was not given antibiotics in the postoperative period. Culture of the lesion yielded no pathogens. The patient's symptoms improved considerably in the early postoperative period and thereafter disappeared completely. The follow-up MRI at 10 months after surgery showed that both the granuloma and the reactive changes at L4-L5 disc space with end-plates level disappeared.

According to Florish et al., ${ }^{[1]}$ post-discectomy MRI reveals changes similar to spondylodiscitis in $10 \%$ of patients. There are also several other reports indicating changes after spondylodiscitis. Our patient had an L4-5 discectomy, and did not have the endplates curetted in the first operation. If the MRI abnormality at L4-5 level had been caused by previous surgery, it would have persisted at the follow-up MRI after resection of the granuloma. We speculated that the disc space changes in our patient were aseptic resulting from inflammation due to remnant bone wax. If these disc space changes had been septic caused by an infectious agent, they would not have disappeared in the follow-up MRI without antibiotic therapy. However, both the granuloma and the "aseptic" changes of L4-5 disc space have disappeared after the operation. We did not perform biopsy studies of the discal material to demonstrate the reactive changes. Therefore, from a scientific perspective, we can only speculate that the changes were secondary to reaction from the wax.

\section{Conclusion}

To our knowledge, this represents the first report revealing the adverse effects of bone wax in spinal surgery. Although bone wax is a safe agent, it may rarely lead to foreign body granuloma and reactive changes in the disc space due to a foreign body reaction in the spine, as shown in this paper. Therefore, bone wax should be used carefully, avoiding its excessive smearing to bone borders because of the risk of causing a foreign body reaction.

\section{References}

1. Floris R, Spallone A, Aref TY, Rizzo A, Apruzzese A, Mulas M, et al. Early postoperative MRI findings following surgery for herniated lumbar disc. Acta Neurochir (Wien) 1997;139:169-75.

2. Curak B, Unal O. Iatrogenic quadriplegia and bone wax. J Neurosurg: (Spine 2) 2000;92:248.

3. Ates O, Cayl? SR, Gurses I bone wax can cause foreign body granuloma in the medulla oblongata. Br J Neurosurg 2004;18:538-40.

4. Patel RB, Kwarter JA, Hodosh RM. Bone wax as a cause of foreign body granuloma in the cerebellopontin angle. J Neurosurg 2000;92:362.

5. Tay HL, Tan LK. Surgical bone wax causing epistaxis. J Laryngol Otol 1996;110:276-78.

6. Low WK, Sim CS. Bone wax foreign body granuloma in the mastoid. ORL J Otorhinolaryngol Relat Spec 2002;64:38-40.

7. Wolvius EB, van der Wal. Bone wax as a cause of a foreign body granuloma in a cranial defect: A case report. Int J Oral Maxillofac Surg 2003;32:656-8

8. Katz SE, Rootman J. Adverse effects of bone wax in surgery of the orbit. Ophtal Plast Reconstr Surg 1996;12:121-6.

9. Verborgt O, Verellen K, Van Thielen F, Deroover M, Verbist L, Borms T. Retroperitoneal tumor as a late complication of the use of bone wax. Acta Orthop Belg 2000;66:389-91.

10. Chun PK, Virmani R, Mason TE, Johnson F. Bone wax granuloma causing saphenous vein graft thrombosis. Am Heart J 1988;115:1310-3.

11. Schonauer C, Tessitore E, Barbagallo G, Albanese V, Moraci A. The use of local agents: Bone wax, gelatin, collagen, oxidized cellulose. Eur Spine J 2004;13:S89-96.

12. Eser O, Cosar M, Aslan A, Sahin O. Bone wax as a cause of foreign body reaction after lumbar disc surgery: A case report. Adv Ther 2007;24:594-7.

13. Sudmann B, Bang G, Sudmann E. Histologically verified bonewax (beeswax) granuloma after median sternotomy in 17 of 18 autopsy cases. Pathology 2006;38:138-41.

14. Anfinsen OG, Sudmann B, Ratt M, Bang G, Sudmann E. Complications secondary to the use of standard bone wax in seven patients. J Foot Ankle Surg 1993;32:505-8

15. Sorrenti SJ, Cumming WJ, Miller D. Reaction of the human tibia to bone wax. Clin Orthop Relat Res 1984;182:293-6.

16. Johnson P, Fromm D. Effects of bone wax on bacterial clearance. Surgery 1992;89:206-9.

17. Finn MD, Schow SR, Schneiderman ED. Osseous regeneration in the presence of four common hemostatic agents. J Oral Maxillofac Surg ;50:608-12.

18. Silber JS, Anderson DG, Vaccaro AR, Anderson PA, McCormick P. NASS Management of postprocedural discitis. Spine J 2002;2:279-87.

Accepted on 03-02-2009

Source of Support: Nil, Conflict of Interest: None declared. 